\title{
Spectrum sharing policy at global level
}

\author{
Marja Matinmikko \\ Centre for Wireless Communications (CWC), University of Oulu, Oulu, Finland, \\ marja.matinmikko@oulu.fi
}

Miia Mustonen

VTT Technical Research Centre of Finland, Oulu, Finland, miia.mustonen@vtt.fi

\begin{abstract}
Spectrum sharing developments exploiting cognitive radio technology will change the traditional spectrum management models, which calls for discussions and decisions in the policy making domain. Efficient governance of natural resources such as the radio spectrum requires actions in different policy making levels ranging from national level all the way to the international level. This Chapter will introduce spectrum sharing related policy making activities in the global level presenting the actions taken at the International Telecommunication Union Radiocommunication sector (ITU-R). We will introduce the groups within ITU-R and their related activities and introduce cognitive radio and spectrum sharing related terminology developed at the ITU-R. Special emphasis is put to the ITU-R studies on cognitive radio systems (CRS) with a set of capabilities for obtaining knowledge, decision making and adjustment, and learning, to enhance the efficiency of spectrum use. We will introduce the CRS capabilities and present scenarios and applications where vertical and horizontal spectrum sharing using CRS capabilities could take place. Other sharing related activities at the ITU-R are also presented including spectrum management, spectrum monitoring and spectrum occupancy measurement studies as well as more general ongoing work on regulatory tools to enable spectrum sharing and CRS from the point view of spectrum management. Finally, a future outlook is given for spectrum sharing policy developments towards the fifth generation $(5 \mathrm{G})$ networks.
\end{abstract}

\section{Introduction}

Research community has made extensive studies on cognitive radio techniques for spectrum sharing to enable several radio systems to operate in the same frequency band on a shared basis. In the early cognitive radio research studies the focus was on technical approaches to detect users with higher priority of spectrum access rights by using spectrum sensing techniques [1] and to select operational channels 
according to some optimization criteria such as interference minimization and throughput maximization [2]. While the early studies on cognitive radio took were about new spectrum management techniques, the link between the cognitive radio techniques developed in the research domain and the actual spectrum management principles adopted in the spectrum regulatory domain remained loose. Thus, the criteria for the development of cognitive radio techniques in the research domain did not thoroughly address the real requirements set for spectrum policy by the regulators and the concerns of involved stakeholders in terms of e.g. interference issues.

As the term 'cognitive radio' very rapidly became commonly used in the research domain in the beginning of the century with various definitions and interpretations, there was a need for a global spectrum policy making body to give guidance on the definition itself and the evolution of the entire cognitive radio topic. While spectrum management and development of spectrum sharing methods and models are a national matter, decided by the local regulatory authorities in the regulatory domain, there is a strong need for coordination between neighboring countries and for harmonization to achieve economies of scale. Therefore, regional and international levels need to develop and promote feasible solutions with potential for wide-spread adoption. The United Nations based International Telecommunication Union Radiocommunication sector (ITU-R) [3-5] plays the key role in spectrum policy making as the truly global body where relevant stakeholders are represented to take decisions on spectrum matters. ITU-R conducts studies on technical and spectrum management aspects, and provides a global forum for discussions and disseminating best practices [3-5].

Since the cognitive radio topics studied and developed in the research domain introduced a novel way of spectrum sharing and coexistence using technical advances to detect other spectrum users and to coordinate interference between different wireless systems in a more dynamic way, there was a need to investigate at the ITU-R whether changes are required in the traditional spectrum management and allocation principles due to the introduction of the cognitive radio concept. This process resulted in a number of studies conducted at the ITU-R and outputs published in several ITU-R documents.

This Chapter will discuss cognitive radio and the related spectrum sharing policy activities at the global level by introducing the relevant activities at the ITU-R and their link to the research domain. By starting with the basic requirements for efficient governance models for natural resources, spectrum sharing related governance principles are first discussed in a high level. Then, the different groups at the ITU$\mathrm{R}$ with activities on cognitive radio and spectrum sharing topics are then summarized including their activities around spectrum sharing and especially cognitive radio systems (CRS) as called by the ITU-R. Finally, a future outlook for the spectrum sharing policy developments are given depicting directions for $5 \mathrm{G}$. 


\section{Spectrum sharing in governance framework}

Management of the radio spectrum belongs to the broader framework of governance of natural resources that have been extensively studied in many fields [6]. The development of effective governance mechanisms is at the heart of managing the precious resources. Efficient management of natural resources requires governance models at different levels ranging from the local level all the way to the global level [6]. Management of the radio spectrum is about defining bundles of property rights over the radio spectrum, and spectrum sharing introduces new bundles of rights that vary with the sharing model [7]. More specifically, the radio spectrum can be seen as a common pool resource (CPR) that is characterized by difficulty to exclude and high subtractability of use [7]. Difficulty to exclude refers to how complicated it is to prevent others from using the same resource. Subtractability of use indicates whether one person's use of the resources diminishes someone else's ability to use the same resources which takes place in the case of the radio spectrum. The guidelines from the management of CPR can provide useful insight into the development of spectrum management and particularly spectrum sharing models.

General principles for robust governance of environmental resources introduces several requirements including providing information, dealing with conflict, inducing rule compliance, providing infrastructure, and being prepared for change [6]. Promising strategies to meet these requirements include dialogue among interested parties, layered institutions including a mix of institutional types, designs that facilitate experimentation, learning, and change, and rules that evolve [6]. Best governance systems in fact are polycentric indicating that there are several points of decision making control that may partially overlap in scope and hierarchy and interact with each other in complex ways that evolve over time [7].

In terms of management of the radio spectrum and particularly introducing dynamic spectrum sharing, efficient governance highlights the different levels of governance for providing information, dealing with conflict, inducing rule compliance, providing infrastructure and readiness for change. Different radio systems that want to use spectrum such as broadcasting, mobile communications, satellite, and fixed and the specific systems within these services, need to be coordinated by mechanisms at various levels to manage the complicated interference scenarios. Thus, spectrum policy discussion and spectrum sharing related activities take place at several levels in the policy making. While the actual awarding of spectrum access rights is done at the national level, spectrum sharing related developments exploiting cognitive radio technologies need international level activities. In the following, we will focus on the global level for spectrum sharing by presenting spectrum sharing developments within the spectrum governance framework at the international level. 


\section{Role of ITU-R}

For spectrum sharing policy making at the global level, the ITU-R plays the key role in the governance framework regarding the global harmonization on spectrum matters. The role of ITU-R is "to ensure the rational, equitable, efficient and economical use of the radio-frequency spectrum by all radiocommunication services, including those using satellite orbits, and to carry out studies and approve Recommendations on radiocommunication matters" [5]. The activities taken at the ITU-R aim at providing an environment that enable the sustainable development of radio communications. In practice this means ensuring interference-free operation of the different radio applications to guarantee quality and reliability of services, harmonization of frequency usage, promotion of new technologies while protecting existing users, and ensuring efficient spectrum utilization. One particularly important part of the ITU-R work is to create conditions for harmonized development and efficient operation of existing and new systems while taking into account the different stakeholders' often conflicting concerns. This becomes a highly complicated task as the ITU-R brings together approximately 40 different radio services that compete for the spectrum allocations. This results in a complex environment for coordinating the various interference scenarios between different services with different technical and operational characteristics.

As being the global body in spectrum policy making, the specific role of the ITU$\mathrm{R}$ is to maintain and extend international cooperation between different countries for the improvement and rational use of telecommunications [3-5]. With this respect the detailed roles taken by the ITU-R as described in [3-5] are to:

-effect allocation of bands of the radio frequency spectrum, the allotment of radio frequencies and the registration of radio frequency assignments and of associated orbital positions in the geostationary satellite orbit in order to avoid harmful interference between radio stations of different countries;

-coordinate efforts to eliminate harmful interference between radio stations of different countries and to improve the use made of radio frequencies and of the geostationary-satellite orbit for radiocommunication services.

-create the regulatory and technical basis for the development and effective operation of satellite and terrestrial climate monitoring and data systems.

Avoidance of harmful interference is a key principle in the above roles of the ITU-R and there are several mechanisms to attain that goal. The global use and management of the radio spectrum requires a high level of international cooperation and an important task of the ITU-R is thus to facilitate the intergovernmental negotiations to develop legally binding agreements between different countries. To ensure interference free operations of the different radio systems, the key tool of the ITU-R is the Radio Regulations (RR) that is the international treaty governing the use of the radio frequency spectrum and satellite orbits. The RR is reviewed and revised regularly through the World Radiocommunication Conferences (WRC) arranged by the ITU-R [8] as shown in Figure 1. The RR defines the recommended 
allocations of frequency bands to the different radio services, and their related technical parameters and procedures for coordination. In preparation for WRCs the ITU$\mathrm{R}$ conducts detailed studies in order to improve the international spectrum regulatory framework with respect to the evolution of existing, emerging and future applications, systems and technologies. The WRC is organized to deal with topics covered by different Agenda Items (AI) and the previous WRC specifies the AIs for the next WRC. These AIs are assigned to different Working Parties (WP) or Task Groups (TG) by Study Groups (SG) where the actual preparatory work is carried out. WPs and TGs are responsible for technical studies for the AIs, such as sharing and coexistence studies between different radiocommunication services when preparing for new spectrum allocations. Detailed sharing considerations related to the specific implementation of the bands are left to the regional and national regulatory bodies. The results of the WRC preparatory studies from the various WPs and TGs are provided to the Conference Preparatory Meeting (CPM) prior to the WRC in the form of draft CPM text. The CPM then prepares a consolidated report on the ITU$\mathrm{R}$ preparatory studies and possible solutions to the WRC AIs.

In addition to maintaining and revising process of RR through the WRCs, ITU$\mathrm{R}$ promotes the development of radio systems and their new technologies to ensure the efficient use of the radio spectrum by studying technical and spectrum management related aspects in various SGs. The results of these studies are published in Reports, Handbooks, and Recommendations. Best practices of spectrum usage are collected and disseminated in workshops, seminars and publications. The current study groups of the ITU-R are [4]:

- SG1: Spectrum management,

- SG3: Radiowave propagation,

- SG4: Satellite services,

- SG5: Terrestrial services,

- SG6: Broadcasting service,

- SG7: Science services.

The Study Groups are presented in more detail in [4]. The key tool for the organization of these studies at the ITU-R is the Radiocommunication Assembly (RA) that is responsible for the structure, programme and approval of radiocommunication studies. RAs are normally convened held every four years and often associated in time and place with WRCs. The RAs determine the Questions to be studied in detail in the different Study Groups of the ITU-R, assign WRC conference preparatory work and other questions to the Study Groups and respond to other requests. RAs also acts as the forum to approve and issue ITU-R Recommendations and Questions developed by the Study Groups, to set the programme for Study Groups and to reshape the Study Group structure. 


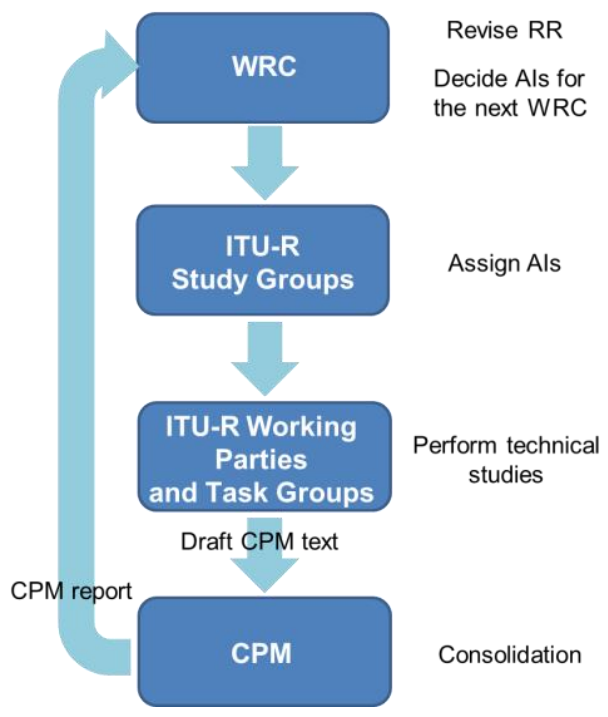

Figure 1. The ITU-R process for revision of RR.

The ITU-R has developed its terminology for the global governance of the radio spectrum to fulfil policy making goals which can differ from the terminology defined and used in the research domain. For example, the ITU-R terminology on spectrum allocations in terms primary and secondary differs from those that were commonly used in the wireless communications research literature on cognitive radios. According to the ITU-R terminology used in the RR [8], "stations of secondary service shall not cause harmful interference to stations of primary services to which frequencies are already assigned or to which frequencies may be assigned at a later date." In addition, they "cannot claim protection from harmful interference from stations of a primary service to which frequencies are already assigned or may be assigned at a later stage". However, "stations of a secondary service can claim protection from harmful interference from stations of the same or other secondary service(s) to which frequencies may be assigned at a later date"[8].

Interference is defined in RR [8] as "the effect of unwanted energy due to one or a combination of emissions, radiations, or inductions upon reception in a radiocommunication system, manifested by any performance degradation, misinterpretation, or loss of information which could be extracted in the absence of such unwanted energy". Harmful interference is interference "which endangers the functioning of a radionavigation service or of other safety services or seriously degrades, obstructs, or repeatedly interrupts a radiocommunication service operating in accordance with Radio Regulations" [8].

The dynamic spectrum sharing work in the form of cognitive radio technology development originally initiated in the research domain has been vetted into the 
ITU-R process of going through studies to become part of the global spectrum regulatory framework. In fact several of the study groups within the ITU-R and the key events (WRC and RA) have been involved in this process. In particular, spectrum sharing using cognitive radio system (CRS) technologies has been studied at the ITU-R in several groups from different perspectives. In the following we present these groups and their activities describing their activities around CRS and dynamic spectrum sharing.

\section{Overview of dynamic spectrum sharing related studies at ITU-R}

Cognitive radio research has considered new innovative ways of accessing the radio spectrum to improve the spectrum utilization efficiency. In the early cognitive radio studies, the system models assumed that the wireless devices themselves could decide on starting to transmit in a frequency channel based on observing the status of the outside world [9]. More specifically, the devices could dynamically share the spectrum by opportunistically accessing channels with the aid of spectrum sensing techniques to protect incumbent primary users by avoiding occupied channels. These approaches made the inherent assumption on the use of spectrum sensing techniques as the mechanism for the protection of incumbent systems from harmful interference. However, the link between what harmful interference meant in the technical studies in the research domain and in the regulatory domain was vague. Thus early on for promoting real-life deployment of the cognitive radio technology there was the need to introduce the cognitive radio principles developed in the research domain into the spectrum regulatory process in the global level.

As a concrete step, the ITU-R decided at its WRC in 2007 (WRC-07) to address the topic of cognitive radio at the following WRC in 2012 (WRC-12). The agenda item 1.19 of WRC-12 was to consider regulatory measures and their relevance, in order to enable the introduction of software-defined radio and cognitive radio systems, based on the results of ITU R studies, in accordance with Resolution 956 (WRC 07). WRC-07 adopted Resolution 956 [10] that invited the ITU-R to study the need for regulatory measures for cognitive radio system (CRS) and software defined radio (SDR). The responsibility on the spectrum management related aspects of CRS was assigned to Study Group 1 and specifically its working party 1B (ITU-R WP1B) while the technical studies on its use particularly in the land mobile service were to be conducted in Study Group 5 by its working party 5A (ITU-R WP5A).

Prior to WRC-07, the RA in 2007 (RA-07) issued Question ITU-R 241 [11] on studies of cognitive radio systems in the mobile service. This question seeked to obtain answers to ITU definition of cognitive radio systems, closely related radio technologies and their functionalities, key technical characteristics, requirements, performance and benefits, potential applications of cognitive radio systems and 
their impact on spectrum management, operational implications, cognitive capabilities that could facilitate coexistence. As the ITU-R Question 241 [11] was specifically focused on the use of cognitive radio systems within the mobile service it was the topic of Study Group 5 and particularly its working party ITU-R WP5A and ITU-R WP5D regarding the land mobile service and IMT systems, respectively.

As a starting point for the preparations for WRC-12, the ITU-R WP1B defined cognitive radio system (CRS) in 2009 in [12] as "A radio system employing technology that allows the system to obtain knowledge of its operational and geographical environment, established policies and its internal state; to dynamically and autonomously adjust its operational parameters and protocols according to its obtained knowledge in order to achieve predefined objectives; and to learn from the results obtained." [12] Technical studies used as a basis for the AI on CRS were conducted in ITU-R WP5A and WP5D and the results of these studies were contained in Reports [13] and [14], respectively. These reports included deployment scenarios, capabilities as well as potential challenges and benefits arising from the cognitive technologies. These three ITU-R reports formed the basis for CPM text for the $\mathrm{AI}$ on cognitive radio for WRC-12.

As a result, the WRC-12 concluded that the introduction of CRSs does not require any changes to the RR and developed a WRC Recommendation on the deployment and use of CRSs [15]. In fact, it was clarified that a CRS is not a radiocommunication service, but rather a system that employs technology that in the future may be implemented in a wide range of applications in the land mobile service. The WRC-12 concluded that any radio system implementing CRS technology needs to operate in accordance with the RR and that the obligations on the protection of stations of other administrations operating in accordance with the RR still hold.

However, it was determined that further studies would be required to explore the potential of cognitive radios. As a result, RA-12 developed Resolution ITU-R 58 [16] regarding further studies on the implementation and use of cognitive radio systems which instructed the ITU-R to continue with the CRS studies. It urged the ITU$\mathrm{R}$ to study operational and technical requirements, characteristics, performance and possible benefits associated with the implementation and use of CRS in relevant radiocommunication services and related frequency bands; to give particular attention to enhancing coexistence and sharing among radiocommunication services [16]. The need for the further studies on CRS presented in ITU-R Resolution 58 [16] was further confirmed at the WRC in the development of WRC Recommendation [15] that recommended administrations to actively participate in the studies. In fact, the resolution triggered a number of studies that are described in more detail in this chapter. The resolution was further updated in 2015.

To conclude the studies on CRS at the ITU-R were based on responding to the topics raised in ITU-Resolution 58 [16] and ITU-R Question 241 [11]. In the course of work, ITU-R Resolution 58 [16] was updated in 2015 after its initial launch in 2012, and ITU-R Question 241 [11] was updated in 2012 and 2015 after its launch in 2007. The spectrum management related work is conducted in Study Group 1 and particularly ITU-R WP1B. While the scope of ITU-R Resolution 58 [16] was 
more generic on the implementation and use of CRS in different radiocommunication services, the focus of Question ITU-R 241 [11] was specific to the mobile service. In fact the technical CRS related work within the ITU-R was mainly focused on the land mobile service while applications to other radiocommunication services are also possible. Thus the technical work was mainly done in Study Group 5. In particular, the ITU-R WP5A developed two reports on CRS in the land mobile service $[13,17]$, and ITU-R WP5D one report on CRS specific to IMT systems [14]. Moreover, other ITU-R Study Groups started to address the topic of CRS from the perspective of being impacted by CRS. For example, ITU-R WP5C studied the impact of CRS on the fixed service and started to prepare a report on it. In Study Group 6, ITU-R WP6A started to study potential interference into broadcasting from CRS devices in the $470-790 \mathrm{MHz}$ band and prepare a report on it but later ended the work.

The cognitive radio studies in the research domain were often motivated by measurements on the spectrum occupancy in different frequency bands in different countries [18]. The spectrum measurement and monitoring related activities within the ITU-R are dealt with in ITU-R WP1C. In fact there were ITU-R studies that are directly related to the measurements conducted by the researchers providing guidelines responding to two ITU-R questions [19-20]. ITU-R Question 233 [19], originally assigned in 2007 and updated in 2011 and extended in 2015, on the measurement of spectrum occupancy. ITU-R Question ITU-R 235 [20] from 2011 and extended in 2015 dealt with spectrum monitoring evolution. It specifically addresses monitoring from the administrations' perspective regarding new considerations for monitoring of radiocommunication systems based on new technologies.

In the following sections we will describe the studies related to CRSs in the mobile service and other related spectrum management studies in more detail.

\section{CRS in Land mobile service}

The technical studies on CRS at the ITU-R have focused on the use of CRS technology within the mobile service in response to ITU-R Question 241 [11]. This section reviews the work done at the ITU-R WP5A that resulted in two published reports on CRS in the land mobile service $[13,17]$. The first report ITU-R Report M.2225 [13] was published in 2011 and it addressed a subset of the questions of the first version of ITU-R Question 241 [11] and provided technical features and capabilities, potential benefits, technical challenges and deployment scenarios for CRS. The second report ITU-R Report M.2330 [17] addressed the updated questions in Question ITU-R 241 [11] from 2012 that asked the ITU-R to study closely related radio technologies and their functionalities, key technical characteristics, requirements, performance improvements and/or other benefits, potential applications of CRS and their impact on spectrum management, how CRS promote efficient use of spectrum, operational implications (including privacy and authentication), CRS 
cognitive capabilities and CRS technologies that could facilitate sharing and coexistence between the mobile service and other services, and factors to be considered for the introduction of CRS technologies in the land mobile service.

As the starting point for the studies, ITU-R WP5A used the ITU-R definition for CRS [12] that identified three key CRS capabilities: 1) obtaining knowledge, 2) decision making and adjustment, and 3) learning. These general capabilities could be applied to different systems in different bands on a case-by case basis and within these capabilities there are several individual techniques. For example, knowledge of spectrum availability for sharing could be obtained via control channels, databases or spectrum sensing techniques. Decision making can take centralized or distributed forms and the parameter to be decided can include frequency channels or power levels for operations among others.

The initial benefits of CRS to operators and end users were depicted in Report ITU-R M.2225 [13]. They include improvements in the efficiency of spectrum use, increased flexibility, self-correction and fault tolerance, resilience in disaster or emergency situation, improved power efficiency, and potential for new mobile communication applications. The report [13] further identified four CRS deployment scenarios:

1. Use of CRS technology to guide reconfiguration of connections between terminals and multiple radio systems,

2. Use of CRS technology by an operator of a radiocommunication system to improve the management of its assigned spectrum resource,

3. Use of CRS technology as an enabler of cooperative spectrum access,

4. Use of CRS technology as an enabler for opportunistic spectrum access in bands shared with other systems and services.

The first two scenarios address an intra-system situation where an operator can use CRS technology to obtain more efficient use of resources within its networks. The latter two are inter-system scenarios that involve spectrum sharing between different operators or systems. In the third scenario, wireless systems collaborate and exchange information about their spectrum use in order to avoid mutual interference. In the fourth scenario, CRSs may access unused spectrum band on a shared basis without causing harmful interference to other systems which resembles the traditional cognitive radio use cases extensively considered in the research domain. Scenarios are also discussed in $[21,22]$.

ITU-R WP5A continued the work in ITU-R Report M.2330 [17] that was published in 2014 providing answers to the remaining topics of the updated ITU-R Question 241 [11]. The report [17] introduced applications of CRS, details of CRS capabilities and enabling technologies, high level characteristics, high level technical and operational requirements, aspects related to CRS performance, potential benefits, factors related to introduction of CRS, and migration issues. 


\section{CRS applications}

ITU-R Report M.2330 [17] specifically addressed spectrum sharing where two or more radio systems operate in the same frequency band as well as coexistence where two or more radio systems operate in adjacent frequency bands. Noting that CRSs could share spectrum with other radio systems that are not necessarily CRSs, as well as with other CRSs, the report introduced two general level spectrum sharing cases:

-Vertical spectrum sharing: The case where one or more radio systems with CRS capabilities share the band of another radio system that does not necessarily have CRS capabilities. The radio systems with CRS capabilities are only allowed to utilize frequencies within the band as long as the other radio system is not affected by harmful interference from the CRSs;

-Horizontal spectrum sharing: The case where multiple radio systems with CRS capabilities are accessing the same shared spectrum band.

Figure 2 illustrates vertical and horizontal spectrum sharing cases where horizontal spectrum sharing refers to systems operating with the same level of access rights while vertical spectrum sharing introduces sharing between systems with different levels of spectrum access rights. Horizontal and vertical spectrum sharing are not mutually existing and both of them can be simultaneously present in various forms in practical applications. Report ITU-R M.2330 [17] identified the following benefits related to vertical and horizontal spectrum sharing:

-Interference minimization: The CRS capability of obtaining knowledge for example using databases can give information on the current protection requirements thus allowing the radio systems to adapt their operations in accordance within the given rules and policies.

-Efficient spectrum use: Additional spectrum can be made available by allowing radio systems to share spectrum with other radio systems leading to increase efficiency of spectrum use which can lead to capacity enhancements for the systems employing CRS technologies.

-Flexible operations: in sharing and coexistence situation CRS system is flexible and could operate over various system configurations and information shared between the involved CRS nodes would ensure that the relevant nodes have the most accurate information of available spectrum in a timely manner. 


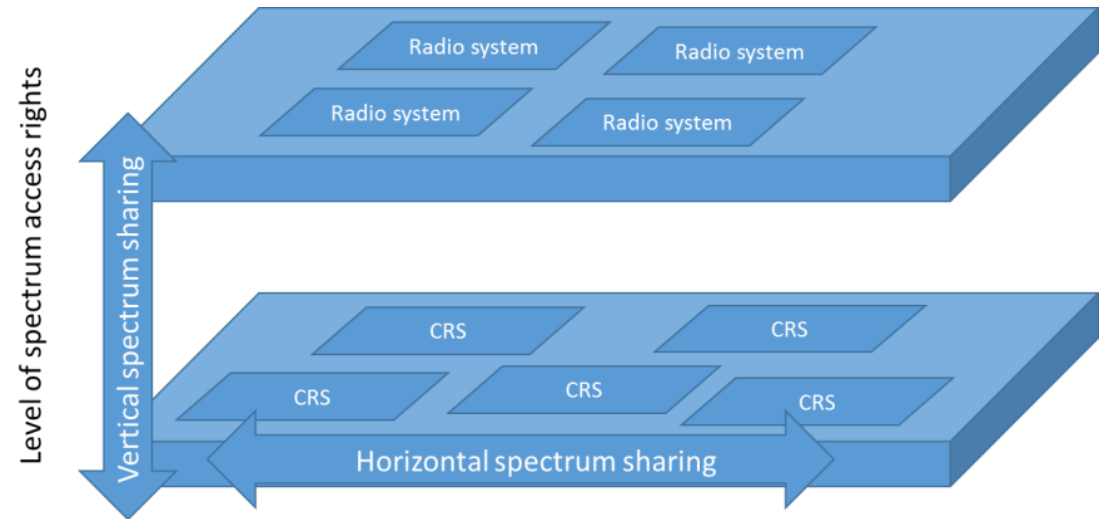

Figure 2. Horizontal and vertical spectrum sharing in the context of CRSs.

The Report ITU-R M.2330 [17] introduced a collection of CRS applications including existing, emerging, and potential applications. An existing application is the radio local area network (RLAN) system operating in the $5250-5350 \mathrm{MHz}$ and $5470-5725 \mathrm{MHz}$ bands on a co-primary basis with radiolocation systems and radar utilizing dynamic frequency selection (DFS) protocol to avoid harmful interference. The RLAN is required to use DFS to ensure by sensing/detection techniques that radiolocation systems are not operating in the same channels and vacate the channels when they appear as described in [23].

An emerging application is the use of TV white space which according to [13] refers to "A portion of spectrum in a band allocated to the broadcasting service and used for television broadcasting that is identified by an administration as available for wireless communication at a given time in a given geographical area on a noninterfering and non-protected basis with regard to other services with a higher priority on a national basis." Some administrations are allowing license-exempt devices to operate on a non-interfering basis in these TV white spaces with the help of CRS capability of geolocation with database access.

Examples of potential CRS applications identified in [17] include cognitive networks exploiting reconfigurable nodes, cognitive mesh networks, heterogeneous system operation using CRS capabilities, intra-system inter-RAT handover, intersystem handover, coordinated spectrum access in heterogeneous radio environment, and vertical and horizontal spectrum sharing enabled by CRS technologies. Several examples of the last application are under study by several administrations to allow additional users to access spectrum with existing incumbent usage with the help of CRS to provide appropriate protection of other radio systems. These examples include Licensed Shared Access in Europe and Citizens Broadband Service (CBRS) in the USA. 


\section{CRS capabilities}

Report ITU-R M.2330 [17] also elaborated the CRS capabilities identified in [12, 13] and provided detailed descriptions of the three key capabilities and their enabling technologies which are depicted in Figure 3. For obtaining knowledge of its operational and geographical environment, established policies and its internal state, the CRS can listen to wireless control channels, use spectrum sensing techniques and/or access databases. Examples of wireless control channels include cognitive control channel (CCC) and cognitive pilot channel (CPC). CCC aims at enhancing coordination between the CRS devices by providing real time communication channel between distributed CRS nodes within a specific geographic area. CPC is a pilot channel that broadcasts radio environment information to CRS devices. Challenges of using wireless control channels for obtaining knowledge of the operational environment include power consumption, synchronization between nodes, contention resolution mechanisms, reliability of information, and strict requirements for timeliness of the data.

Spectrum sensing is another enabling technique identified in [17] for obtaining knowledge and has been widely studied in the cognitive radio research $[1,2,9]$. Spectrum sensing is the capability to detect other signals around the CRS node. There are different sensing techniques with varying sensing capabilities, requirements for a priori information, and degrees of complexity. Moreover, the use of sensing techniques can be with single or multiple devices and by CRS nodes themselves or by as dedicated listening devices or community sensor networks. There are several challenges related to the use of spectrum sensing for obtaining knowledge or the operational environment including hidden node problem, reliability of sensing, implementation of particularly wideband sensing, power and processing consumption, signaling cost, performance in realistic settings, and detection of receive only nodes. The report discussed that the implementation of opportunistic spectrum access could not rely solely on spectrum sensing techniques but would additionally require alternative methods.

The third technique for obtaining knowledge in [17] is the use of databases often combined with geolocation capability where the CRS node knows its location. CRS nodes can access a database that provides information about the locally usable frequencies ensuring that incumbent services remain protected from harmful interference. Databases can provide information of vacant frequency channels as well as the rules related to the use of the channels in certain locations. Many of the CRS applications introduced previously such as TV white space, LSA and CBRS have taken the database approach. The implementation can take various forms such as single open database, multiple open databases, proprietary close databases, or clearinghouse that aggregates and host raw data from multiple providers. Definition of open interfaces and protocols is important for allowing different types of CRS nodes to access different databases. Challenges for the database approach with geolocation include e.g. timely update of data, availability of information about the stations to be protected, and security and privacy aspects. 


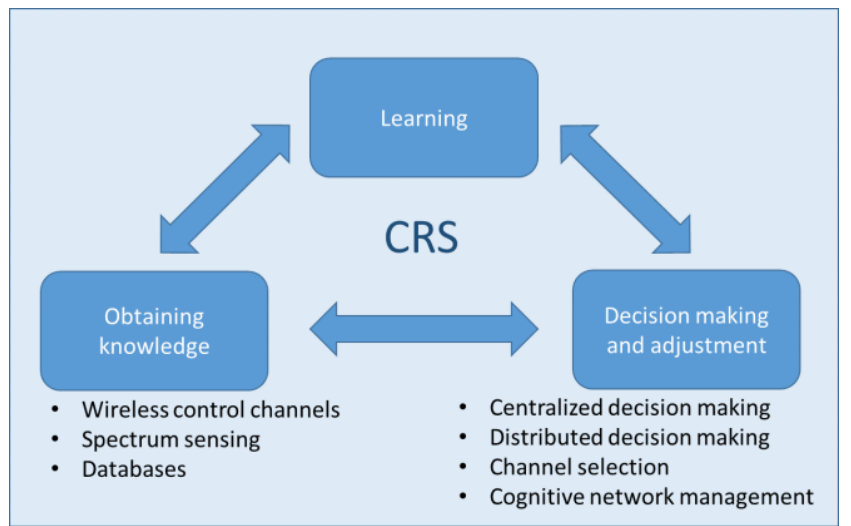

Figure 3. CRS capabilities and enabling technologies.

The CRS capability of decision making and adjustment of operational parameters and protocols (see Figure 3) discussed in Report ITU-R M.2330 [17] involves the CRSs to take actions in link level and network level that take into account underlying policies in the dynamic operational environment. The report identifies centralized and distributed decision making techniques and provides examples of channel selection and cognitive network management for CRS in the land mobile service. Centralized decision making assumes a centralized entity for decision making that informs the CRS nodes about adaptations of operational parameters such as spectrum resources. Distributed decision making is based on localized decisions of distributed CRS nodes which can involve coordination between nodes. In the case of spectrum sharing, decision making can involve the selection of channels for operation. Moreover, dynamic adjustment of reconfigurable nodes in a network, cognitive network management functions are needed.

Finally, learning as a CRS capability can enable performance improvement by allowing the CRS to use stored information of its own actions and other's actions and the results of the actions to help in the decision making process. Learning can can make the operations of the CRS more efficient by e.g. improving fault tolerance, learning traffic patterns in different frequency channels for channel selection, and enhancing network management to adjust to different requirements.

\section{CRS characteristics, requirements and performance aspects}

CRS introduces new high level characteristics over conventional radio systems including aspects related to flexible spectrum management in temporal, frequency and geographical domains to improve spectrum efficiency and dynamic coordination among radio systems to facilitate spectrum sharing and coexistence [17]. More accurate knowledge of the characteristics and operations of other systems combined 
with dynamic interference management could help the CRSs to avoid causing harmful interference and share the spectrum more efficiently in horizontal and vertical spectrum sharing cases.

New requirements for CRSs over traditional radio systems arise from CRS internal operations as well as from interactions with other systems operating in the same channel or adjacent channel in spectrum sharing and coexistence cases, respectively. Horizontal and vertical spectrum sharing and coexistence with other systems set requirements on the CRSs requesting them to support specific technical features and functionalities to avoid harmful interference to other radio systems with same level or higher level of spectrum usage rights in dynamic conditions where the spectrum use of the channels changes.

CRSs that introduce radio operations with dynamic availability of spectrum call for new metrics to characterize the system internal performance, interference considerations in spectrum sharing and coexistence between radio systems, and overall spectrum usage efficiency. Initial benefits from [13] are expanded in [17] specifically to vertical and horizontal spectrum sharing cases which were discussed above. In addition, CRS can provide benefits for operators by introducing dynamic spectrum reconfiguration, radio resource optimization, and dynamic device context provision. Finally, introduction of CRS capabilities into the land mobile service introduces migration aspects which were also discussed in [17] including inclusion of information exchange between different systems to facilitate spectrum sharing.

\section{CRS for IMT systems}

International Mobile Telecommunications (IMT) systems, the ITU-R terminology encompassing the mobile communication networks from $3 \mathrm{G}$ to upcoming $5 \mathrm{G}$, are addressed in ITU-R WP5D. While majority of the studies on cognitive radio techniques at the ITU-R was focused on mobile communication systems in response to ITU-R Question 241 [11] encompassing both IMT and non-IMT systems, the IMT specific work on spectrum sharing and CRS remained limited. In fact in the research domain, a lot of effort was put on developing cognitive radio techniques for cellular systems where spectrum sharing was assumed between operators or between cellular systems and other systems. However, the work at the ITU-R on CRS specific for IMT systems resulted in only one report ITU-R M.2242 developed by the ITU-R WP5D in 2011 [14]. The report studied the impact of adding CRS capabilities to existing IMT systems, and analysed the benefits, challenges and impacts of CRSs in IMT, particularly regarding the impact on the use of IMT spectrum. The report emphasized that an IMT system employing CRS technology should still meet the minimum requirements for IMT systems and that that the existing IMT systems should not suffer from harmful interference and quality-of-service (QoS) degradation from the introduction of CRS technology.

The report [14] took a very cautious view on what CRS could mean for IMT systems and only focused on single-operator scenarios where an operator would use 
CRS technology to enhance its own performance as the exclusive owner of the spectrum. The report preferred the operator centric intra-operator approach as the scenario for IMT to benefit from some CRS capabilities. It is restricted to improvement in the spectrum usage efficiency by accessing spectrum resources from one IMT system for other IMT systems inside the domain of a single operator. The report [14] identified the following scenarios for CRS in IMT systems:

1. Update of a network for optimized radio resource usage,

2. Upgrade of an existing radio interface or a network with a new radio interface,

3. In-band coverage/capacity improvement by relays,

4. Self-configuration and self-optimization of femtocells,

5. Multi-modes coexistence and simultaneous transmission.

The intra-operator scenarios involved cases where an operator who is the exclusive owner of the spectrum may use cognitive radio features to better manage its heterogeneous radio access networks. CRS scenarios for IMT are also discussed in [24].

The report identified benefits from CRS in IMT in the intra-operator case and in terms of overall spectrum efficiency and capacity improvement, radio resources utilization flexibility, and interference mitigation. The report concluded that the introduction of CRS in IMT systems in intra-operator case is the preferred scenario which did not include vertical or horizontal spectrum sharing. Even in that case, a concern is to ensure that existing radio systems do not suffer from harmful interference or QoS degradation from CRS technology, which shows the caution of the operators about the topic.

There has been a lot of work at the ITU-R on sharing and compatibility studies between IMT systems and other radiocommunication systems for the potential use of IMT systems in the bands currently used by other services. These studies take into account the characteristics of the IMT systems and the other systems to identify the potential interferences between the systems. They typically identify for protection criteria in terms of e.g. separation distance, transmission power limit, interference mitigation techniques, and feasibility of sharing. These studies typically characterize the static situations without the use of CRS capabilities and dynamic spectrum sharing as such. To some extend they have taken into account the use of interference mitigation techniques which has seen to reduce the protection distances. In fact the CRS capabilities could help in the sharing studies to relax the conditions and improve opportunities for sharing and coexistence between different radio systems.

The most recent IMT developments are now focusing on the fifth generation (5G) of mobile communication systems denoted as IMT-2020 at the ITU-R. The IMT the work at the ITU-R is specifically addressing the preparations for WRC-19 AI 1.13 about the possible new spectrum allocations for IMT systems in the frequency range between $24-86 \mathrm{GHz}$. 


\section{Spectrum management related studies at ITU-R}

While the technical studies on CRS were conducted in SG5 of the ITU-R, spectrum management related aspects of CRS belong to SG1. The CRS related spectrum measurement studies and new spectrum management principles studied at SG1 are reviewed next.

\section{Spectrum measurements and occupancy studies}

Many of the cognitive radio studies in the research domain were motivated by spectrum measurement studies conducted by research people where the spectrum occupancies of different frequency bands were analyzed as summarized in [18]. In fact a number of spectrum measurement campaigns were conducted around the world to quantify the percentage of time that the received signal levels exceed some threshold giving an indication of the channel occupancies. The main findings from the research studies indicated very low levels of spectrum occupancy in many bands which gave motivation for the studies to introduce dynamic spectrum sharing in bands with low occupancy levels. While these measurements were mainly done by the research community, spectrum measurement and monitoring had been a standard tool in the spectrum regulatory domain for a long time. However, the basic guidelines developed in the regulatory domain were not applied to a large extent in the research domain.

At the same time as the CRS studies were conducted at the ITU-R, there were studies about the measurement of spectrum occupancy and spectrum monitoring that took place in Study Group 1. In fact, studies addressed two ITU-R Questions, ITU-R Question 233 [19] about measurement of spectrum occupancy and ITU-R Question ITU-R 235 [20] on spectrum monitoring evolution. ITU-R Question 233 [19] originated from RA-07 and was updated in 2011 and expanded in 2015. It called for identifying techniques to perform frequency channel and frequency band occupancy measurements including processing and presentation methods. It also seeked to define the term "occupancy" for frequency channel and frequency band measurements, and defining and applying threshold levels in practical situations. Question ITU-R 235 [20] initiated in 2011 was extended in 2015 focused on spectrum monitoring evolution with the advent of new technologies. It looked for new considerations for monitoring of radiocommunication systems including new approaches in terms of organisation, procedures and equipment to monitor systems based on future radiocommunication technologies. It is particularly concerned with the administrations positions and seeks for the needs for administrations in order to implement the new approaches to monitor systems based on future radiocommunication technologies.

The studies have resulted in several reports and recommendations published by the ITU-R [25-28]. Recommendation ITU-R SM.2039 [25] on spectrum monitoring 
evolution published in 2013 provides answers to Question ITU-R 235 [20] and recommends new technologies to be used in spectrum monitoring evolution to extend monitoring coverage including detection of weak signals, co-frequency signal separation, and multi-mode location based on a combination of techniques. Report ITU-R SM.2355 [26] published in 2015 highlights the role of spectrum monitoring as an important tool in the management of radio spectrum by providing monitoring data, including spectrum occupancy, characteristic of signal, such as field strength, bandwidth, modulation type and location of emitter, etc. The report addresses the challenges of future spectrum monitoring systems that should have capability for monitoring new radiocommunication technologies and systems including those with CRS capabilities, such as detection of weak signal, co-frequency signal separation and multi-mode location based on digital signal processing and network. The report also provides examples of advanced monitoring techniques.

Recommendation ITU-R SM.1880 [27] on spectrum occupancy measurement and evaluation originally published in 2011 and revised in 2015 specifies the measurement procedures and techniques in response to Question ITU-R 233 [19]. It recommends the use of Report ITU-R SM.2256 [28] and ITU-R Handbook on spectrum monitoring [29] to be used as guidance and equipment should fulfil the requirements set in those documents. Report ITU-R SM.2256 [28] on spectrum occupancy measurement and evaluation was published in 2012 and further updated in 2016. The report provides a comprehensive description of spectrum occupancy measurement terminology and methodology and acts as the guideline for conducting spectrum occupancy measurements. The report includes relevant terminology, measurement parameters, measurement procedure, calculation of occupancy, presentation of results, as well as interpretation and use of results. Finally, the ITUR Handbook on spectrum monitoring [29] whose latest edition is from 2011 contains ITU-R guidelines for monitoring.

\section{New spectrum management principles}

In ITU-R, the non-technical spectrum management related issues are handled in the WP 1B "Spectrum management methodologies and economic strategies" of SG1. The WP1B considers for example spectrum management fundamentals, methodologies as well as both national and international regulatory frameworks. The studies performed in this WP are done in a technology and service neutral manner. After completing of the preparatory work for WRC-12 on CRS, the WP1B is currently working on two reports regarding more dynamic spectrum use. Similarly to prior studies on the CRS, the first report is being developed in response to ITU-R Resolution 58 [16]. This current working document towards a preliminary draft new (PDN) report on spectrum management challenges [30] is addressing spectrum management principles and spectrum engineering techniques for the dynamic access to spectrum by radio system employing CRS capabilities. Whereas the previous 
CRS reports were service specific and concentrated on the technical aspects of CRS developed in ITU-R WP5A and ITU-R WP5D, the focus of this report is to study general framework and highlight some challenges related to CRS techniques including the means to ensure the protection of incumbent services sharing the same band or operating in the adjacent bands.

The second working document towards a PDN Report on innovative regulatory tools [31] is being developed within the framework of an ITU-R Question 208-1/1 [32] "Alternative methods of national spectrum management". This report aims to provide a collection of regulatory mechanisms which may be implemented on a national basis that have been experimented and are recognized as best practices in terms of spectrum management solutions by administrations. Currently the draft report includes the European LSA approach and an approach to share spectrum between multiple mobile operators.

\section{Conclusions and future outlook}

At the international level, the ITU-R has developed general guidelines for dynamic spectrum sharing using the CRS technology. These guidelines form the ground for spectrum sharing in the global scale. The main conclusions of the CRS work for WRC-12 was that the introduction of CRS does not require any changes to the RR and that a system employing CRS technology needs to operate in accordance with the RR.

As defined by the ITU-R, the CRS is a set of capabilities for obtaining knowledge, decision making and adjustment, and learning, which can be applied to different radiocommunication services. The CRS work within the ITU-R mainly focused on the land mobile service while applications to other radiocommunication services are also seen possible. The ITU-R introduced vertical and horizontal spectrum sharing applications where the distinction comes from the different levels of spectrum usage rights between the wireless systems operating in the same spectrum band.

In fact the ITU-R guidelines for CRS have been there for a few years now. ITU$\mathrm{R}$ studies on CRS capabilities expanded the considerations from pure spectrum sensing techniques to wireless control channels and databases. In the research domain the interest in pure cognitive radio technology has shifted to the development of specific spectrum sharing models that exploit CRS capabilities. At the same time these sharing models have been introduced at the ITU-R level to exchange information about new spectrum management approaches. The role of the ITU-R continues to be the global forum for gathering and disseminating best practices, providing place for discussions by all stakeholders, and conducting studies of technical and spectrum management aspects.

The development of fifth generation (5G) networks is a major on-going effort in research, industry and regulatory domains regarding future mobile communication systems. While the prior work carried out in the scope of on CRS specific to the 
IMT systems at the ITU-R remained limited and focused only on intra-operator scenarios, spectrum sharing will have a more prominent role in $5 \mathrm{G}$. In the future, spectrum sharing will find new applications areas in the development and deployment of $5 \mathrm{G}$ networks. $5 \mathrm{G}$ networks are envisaged to be deployed in a wide variety of frequency bands ranging from the existing bands with mobile allocation towards higher frequency range between 24-86 Ghz studied for WRC-19. In the context of $5 \mathrm{G}$, spectrum sharing in vertical and horizontal dimensions will play an increasingly important role to allow efficient utilization of the spectrum for the different stakeholders. Operations in these higher frequencies will call for new models and techniques for both horizontal and vertical spectrum sharing to allow local deployments and to protect possible incumbents, respectively.

\section{References}

[1] Yücek T, Arslan H (2009) A survey of spectrum sensing algorithms for cognitive radio applications. IEEE Communication Surveys and Tutorials 11(1): 116-130

[2] Akyildiz I F, Lee WY, Vuran MC, Mohanty S (2008) A survey on spectrum management in cognitive radio networks. IEEE Communications magazine 46: 40-48

[3] ITU-R (2014) ITU-R Radiocommunication. Committed to connecting the world. International Telecommunications Union Radiocommunication sector. Available at http://www.itu.int/dms_pub/itu-r/opb/gen/R-GEN-OVW-2014-PDF-E.pdf

[4] ITU-R (2013) ITU-R study group booklet. International Telecommunications Union Radiocommunication sector. Available at http://www.itu.int/dms pub/itu-r/opb/gen/R-GEN-SGB2013-PDF-E.pdf

[5] ITU-R (2017) Welcome to the ITU-R. International Telecommunications Union Radiocommunication sector. Available at http://www.itu.int/en/ITU-R/information/Pages/default.aspx

[6] Dietz T, Ostrom E, Stern PC (2003) The struggle to govern the commons. Science 302: 19071912.

[7] Weiss MBH, Lehr WH, Acker A, Gomez MM (2015) Socio-technical considerations for Spectrum Access System (SAS) design. IEEE International Symposium on Dynamic Spectrum Access Networks (DySPAN), Stockholm, pp. 35-46.

[8] ITU (2016) The Radio Regulations. International Telecommunications Union Radiocommunication sector.

[9] Haykin S (2005). Cognitive radio: brain-empowered wireless communications. IEEE journal on selected areas in communications 23: 201-220

[10] ITU-R (2007) Regulatory measures and their relevance to enable the introduction softwaredefined radio and cognitive radio systems. Resolution 956 (WRC-07). International Telecommunication Union Radiocommunication sector.

[11] ITU-R (2015) Cognitive radio systems in the mobile service. ITU-R Question 241-3/5. International Telecommunication Union Radiocommunication sector.

[12] ITU-R (2009) Definitions of software-defined radio (SDR) and cognitive radio system (CRS). Report ITU-R SM.2152. International Telecommunications Union Radiocommunication sector.

[13] ITU-R (2011) Introduction to cognitive radio systems in the land mobile service. Report ITUR M.2225. International Telecommunications Union Radiocommunication sector.

[14] ITU-R (2011) Cognitive radio systems specific for International Mobile Telecommunications systems. Report ITU-R M.2242. International Telecommunications Union Radiocommunication sector.

[15] ITU-R (2012) Deployment and use of cognitive radio systems. Recommendation 76 (WRC12). International Telecommunications Union Radiocommunication sector. 
[16] ITU-R (2012) Studies on the implementation and use of cognitive radio systems. ITU-R Resolution 58. International Telecommunications Union Radiocommunication sector.

[17] ITU-R (2014) Cognitive radio systems in the land mobile service. Report ITU-R M.2330. International Telecommunications Union Radiocommunication sector, Geneva, Switzerland.

[18] Höyhtyä M, Mämmelä A, Eskola M, Matinmikko M, Kalliovaara J, Ojaniemi J, Suutala J, Ekman R, Bacchus R, Roberson D (2016) Spectrum occupancy measurements: A survey and use of interference maps. IEEE Communications Surveys \& Tutorials 18: 2386-2414

[19] ITU-R (2011) Question ITU-R 233-1/1. International Telecommunications Union Radiocommunication sector.

[20] ITU-R (2011) Spectrum monitoring evolution. Question ITU-R 235/1. International Telecommunications Union Radiocommunication sector.

[21] Mustonen M, Matinmikko M (2014) Scenarios for CRS from ITU-R. In A. Medeisis \& O. Holland (eds.) Cognitive Radio Policy and Regulation: Techno-Economic Studies to Facilitate Dynamic Spectrum Access. Springer.

[22] Filin S, Murakami H, Harada H, Yoshino H, Kashiki K, Shibata T (2011) ITU-R standardization activities on cognitive radio systems. 6th International ICST Conference on Cognitive Radio Oriented Wireless Networks and Communications (CROWNCOM), Osaka, pp. 116120.

[23] ITU-R (2011) Dynamic frequency selection in wireless access systems including radio local area networks for the purpose of protecting the radiodetermination service in the $5 \mathrm{GHz}$ band. Recommendation ITU-R M. 1652-1. International Telecommunications Union Radiocommunication sector.

[24] Sayrac B, Uryga H, Bocquet W, Cordier P, Grimoud S (2013) Cognitive radio systems specific for IMT systems: Operator's view and perspectives. Telecommunications Policy 37: 154166

[25] ITU-R (2013) Spectrum monitoring evolution. Recommendation ITU-R SM.2039. International Telecommunications Union Radiocommunication sector.

[26] ITU-R (2015) Spectrum monitoring evolution. Report ITU-R SM.2355. International Telecommunications Union Radiocommunication sector.

[27] ITU-R (2015) Spectrum occupancy measurement and evaluation. Recommendation ITU-R SM.1880-1. International Telecommunications Union Radiocommunication sector.

[28] ITU-R (2016) Spectrum occupancy measurements and evaluation. Report ITU-R SM.22561. International Telecommunications Union Radiocommunication sector.

[29] ITU-R (2011) Handbook on spectrum monitoring. International Telecommunications Union Radiocommunication sector.

[30] ITU-R (2016) Working Document toward PDNR SM.[CRS SPECTRUM MANAGEMENT CHALLENGES]. International Telecommunication Union Radiocommunication sector.

[31] ITU-R (2016) Working Document toward PDNR SM.[REGULATORY TOOLS]. International Telecommunication Union Radiocommunication sector.

[32] ITU-R (2015) Alternative methods of national spectrum management. ITU-R Question 2081/1. International Telecommunications Union Radiocommunication sector. 